\title{
Asymptotic Stability of Fractional Stochastic Neutral Differential Equations with Infinite Delays
}

\author{
R. Sakthivel, ${ }^{1}$ P. Revathi, ${ }^{2}$ and N. I. Mahmudov ${ }^{3}$ \\ ${ }^{1}$ Department of Mathematics, Sungkyunkwan University, Suwon 440-746, Republic of Korea \\ ${ }^{2}$ Department of Mathematics, Anna University of Technology, Coimbatore 641 047, India \\ ${ }^{3}$ Department of Mathematics, Eastern Mediterranean University, Gazimagusa, Mersin 10, Turkey
}

Correspondence should be addressed to R. Sakthivel; krsakthivel@yahoo.com

Received 11 September 2012; Accepted 8 November 2012

Academic Editor: Elena Braverman

Copyright (c) 2013 R. Sakthivel et al. This is an open access article distributed under the Creative Commons Attribution License, which permits unrestricted use, distribution, and reproduction in any medium, provided the original work is properly cited.

\begin{abstract}
We study the existence and asymptotic stability in $p$ th moment of a mild solution to a class of nonlinear fractional neutral stochastic differential equations with infinite delays in Hilbert spaces. A set of novel sufficient conditions are derived with the help of semigroup theory and fixed point technique for achieving the required result. The uniqueness of the solution of the considered problem is also studied under suitable conditions. Finally, an example is given to illustrate the obtained theory.
\end{abstract}

\section{Introduction}

The stochastic differential equations have been widely applied in science, engineering, biology, mathematical finance and in almost all applied sciences. In the present literature, there are many papers on the existence and uniqueness of solutions to stochastic differential equations (see [1-4] and references therein). More recently, Chang et al. [5] investigated the existence of square-mean almost automorphic mild solutions to nonautonomous stochastic differential equations in Hilbert spaces by using semigroup theory and fixed point approach. $\mathrm{Fu}$ and Liu [2] discussed the existence and uniqueness of square-mean almost automorphic solutions to some linear and nonlinear stochastic differential equations and in which they studied the asymptotic stability of the unique squaremean almost automorphic solution in the square-mean sense. On the other hand, recently fractional differential equations have found numerous applications in various fields of science and engineering [6]. The existence and uniqueness results for abstract stochastic delay differential equation driven by fractional Brownian motions have been studied in [7]. In particular the stability investigation of stochastic differential equations has been investigated by several authors [8-15].

Let $K$ and $H$ be two real separable Hilbert spaces with inner products $\langle\cdot, \cdot\rangle_{K}$ and $\langle\cdot, \cdot\rangle_{H}$, respectively. We denote their norms by $|\cdot|_{K}$ and $|\cdot|_{H}$. To avoid confusion we just use $\langle\cdot, \cdot\rangle$ for the inner product and $|\cdot|$ for the norm. Let $\left\{e_{i}\right\}_{i=1}^{\infty}$ be an orthonormal basis of $K$. Throughout the paper, we assume that $(\Omega, \mathscr{F}, P ; \mathbb{F})\left(\mathbb{F}=\left\{\mathscr{F}_{t}\right\}_{t>0}\right)$ is a complete filtered probability space satisfying that $\mathscr{F}_{0}$ contains all $P$ null sets of $\mathscr{F}$. Suppose $\{W(t): t \geq 0\}$ is cylindrical $K$ valued Brownian motion with a trace class operator $Q$, denote $\operatorname{trac} Q=\sum_{i=1}^{\infty} \lambda_{i}=\lambda<\infty$, which satisfies that $Q e_{i}=\lambda_{i} e_{i}$. So, actually, $W(t)=\sum_{i=1}^{\infty} \sqrt{\lambda_{i}} W_{i}(t) e_{i}$, where $\left\{W_{i}(t)\right\}_{i=1}^{\infty}$ are mutually independent one-dimensional standard Brownian motions. Define $\mathscr{L}(K, H)$ as the set of all bounded linear operators $A: K \rightarrow H$ with the following norm:

$$
|A|=\left(\sum_{i=1}^{\infty}\left|A e_{i}\right|^{2}\right)^{1 / 2}<\infty .
$$

It is obvious that $\mathscr{L}(K, H)$ is a Hilbert space with an inner product induced by the above norm. Let $A \in \mathscr{L}(K, H)$ be called a Hilbert-Schmidt operator. We further assume that the filtration is generated by the cylindrical Brownian motion $W(\cdot)$ and augmented, that is,

$$
\mathscr{F}_{t}=\sigma\{W(s) ; 0 \leq s \leq t\} \vee \mathcal{N},
$$

where $\mathcal{N}$ is the $P$-null sets. 
The qualitative properties of stochastic fractional differential equations have been considered only in few publications. El-Borai et al. [16] studied the existence uniqueness, and continuity of the solution of a fractional stochastic integral equation. Ahmed [17] derived a set of sufficient conditions for controllability of fractional stochastic delay equations by using a stochastic version of the well-known Banach fixed point theorem and semigroup theory. Moreover, theory of neutral differential equations is of both theoretical and practical interests. For a large class of electrical networks containing lossless transmission lines, the describing equations can be reduced to neutral differential equations. However, to the author's best knowledge no work has been reported in the present literature regarding the existence, uniqueness, and asymptotic stability of mild solutions for neutral stochastic fractional differential equations with infinite delay in Hilbert spaces. Motivated by this consideration, in this paper we consider the nonlinear fractional neutral stochastic differential equations with infinite delays in the following form:

$$
\begin{aligned}
& { }^{c} D_{t}^{\alpha}[X(t)+g(t, X(t-\tau(t)))] \\
& =A X(t)+f(t, X(t-\tau(t))) \\
& \quad+\sigma(t, X(t-v(t))) \frac{d W(t)}{d t}, \quad t \geq 0, \\
& \quad X_{0}(\cdot)=\varphi \in \mathscr{B}_{\mathscr{F}}([m(0), 0], H),
\end{aligned}
$$

where $A$ is the infinitesimal generator of a strongly continuous semigroup of a bounded linear operator $S(t), t \geq 0$ in the Hilbert space $H, f: R_{+} \times H \rightarrow H, \sigma: R_{+} \times H \rightarrow \mathscr{L}(K, H)$ are two Borel measurable mappings, and $g: R_{+} \times H \rightarrow H$ is continuous mapping. The fractional derivative ${ }^{c} D_{t}^{\alpha}, \alpha \in$ $(0,1)$ is understood in the Caputo sense. In addition, let $\tau(t), v(t) \in C\left(R_{+}, R_{+}\right)$satisfy $t-\tau(t) \rightarrow \infty, t-v(t) \rightarrow \infty$ as $t \rightarrow \infty$. Let $m(0)=\max \left\{\inf _{s \geq 0}(s-\tau(s)), \inf _{s \geq 0}(s-\right.$ $v(s))\}$. Here $\mathscr{B}_{\mathscr{F}_{0}}([m(0), 0], H)$ denote the family of all almost surely bounded, $\mathscr{F}_{0}$-measurable, continuous random variables $\varphi(t):[m(0), 0] \rightarrow H$ with norm $|\varphi|_{\mathscr{B}}=$ $\sup _{m(0) \leq t \leq 0} E|\varphi(t)|_{H}$. Throughout this paper, we assume that $p \geq 2$ is an integer.

\section{Preliminaries and Basic Properties}

Let $A$ be the infinitesimal generator of an analytic semigroup $S(t)$ in $H$. Then, $(A-\eta I)$ is invertible and generates a bounded analytic semigroup for $\eta>0$ large enough. Therefore, we can assume that the semigroup $S(t)$ is bounded and the generator $A$ is invertible. It follows that $(-A)^{\eta}, 0<\eta \leq 1$ can be defined as a closed linear invertible operator with its domain $D(-A)^{\eta}$ being dense in $H$. We denote by $H_{\eta}$ the Banach space $D(-A)^{\eta}$ endowed with the norm $|x|_{\eta}=\left|(-A)^{\eta} x\right|$, which is equivalent to the graph norm of $(-A)^{\eta}$. For more details about semigroup theory, one can refer [18].

Lemma 1 (see [18]). Suppose that the preceding conditions are satisfied.

(a) Let $0<\eta \leq 1$, then $H_{\eta}$ is a Banach space. (b) If $0<v \leq \eta$, then the embedding $H_{v} \subset H_{\eta}$ is compact whenever the resolvent operator of $A$ is compact.

(c) For every $\eta \in(0,1]$, there exists a positive constant $C_{\eta}$ such that $\left\|A^{\eta} S(t)\right\| \leq C_{\eta} / t^{\eta}, t>0$.

Definition 2 (see [19]). The fractional integral of order $q$ with the lower limit 0 for a function $f$ is defined as

$$
I^{\alpha} f(t)=\frac{1}{\Gamma(\alpha)} \int_{0}^{t} \frac{f(s)}{(t-s)^{1-\alpha}} d s, \quad t>0, q>0
$$

provided the righthand side is pointwise defined on $[0, \infty)$, where $\Gamma(\cdot)$ is the gamma function.

Definition 3 (see [19]). The Caputo derivative of order $\alpha$ for a function $f:[0, \infty) \rightarrow R$ can be written as

$$
\begin{array}{r}
{ }^{c} D^{\alpha} f(t)=\frac{1}{\Gamma(n-\alpha)} \int_{0}^{t} \frac{f^{(n)}(s)}{(t-s)^{\alpha+1-n}} d s=I^{n-\alpha} f^{(n)}(t), \\
t>0,0 \leq n-1<\alpha<n .
\end{array}
$$

If $f$ is an abstract function with values in $H$, then integrals which appear in the above definitions are taken in Bochner's sense.

According to Definitions 2 and 3, it is suitable to rewrite the stochastic fractional equation (3) in the equivalent integral equation

$$
\begin{aligned}
& X(t)=[\varphi(0)+g(0, \varphi)]-g(t, X(t-\tau(t))) \\
&+\frac{1}{\Gamma(\alpha)} \int_{0}^{t}(t-s)^{\alpha-1}[A X(s)+f(s, X(s-\tau(s))) \\
&\left.+\sigma(s, X(s-v(s))) \frac{d W(s)}{d s}\right] d s .
\end{aligned}
$$

In view of [18, Lemma 3.1] and by using Laplace transform, we present the following definition of mild solution of (3).

Definition 4. A stochastic process $\{X(t): t \in[0, T]\}, 0 \leq$ $T<\infty$ is called a mild solution of (3), if

(i) $X(t)$ is $\mathscr{F}_{t}$-adapted and is measurable, $t \geq 0$;

(ii) $X(t) \in H$ has càdlàg paths on $t \in[0, T]$ almost surely and for each $t \in[0, T]$, the function $(t-s)^{\alpha-1} A T_{\alpha}(t-$ $s) g(s, X(s-\tau(s)))$ is integrable such that the following integral equation is satisfied:

$$
\begin{aligned}
X(t)= & S_{\alpha}(t)[\varphi(0)+g(0, \varphi)]-g(t, X(t-\tau(t))) \\
& -\int_{0}^{t}(t-s)^{\alpha-1} A T_{\alpha}(t-s) g(s, X(s-\tau(s))) d s \\
& +\int_{0}^{t}(t-s)^{\alpha-1} T_{\alpha}(t-s) f(s, X(s-\tau(s))) d s \\
& +\int_{0}^{t}(t-s)^{\alpha-1} T_{\alpha}(t-s) \sigma(s, X(s-v(s))) d W(s),
\end{aligned}
$$


(iii) $X_{0}(\cdot)=\varphi \in \mathscr{B}_{\mathscr{F}}([m(0), 0], H)$,

where

$$
\begin{gathered}
S_{\alpha}(t) X=\int_{0}^{\infty} \eta_{\alpha}(\theta) S\left(t^{\alpha} \theta\right) X d \theta, \\
T_{\alpha}(t) X=\alpha \int_{0}^{\infty} \theta \eta_{\alpha}(\theta) S\left(t^{\alpha} \theta\right) X d \theta
\end{gathered}
$$

with $\eta_{\alpha}$ a probability density function defined on $(0, \infty)$.

The following properties of $S_{\alpha}(t)$ and $T_{\alpha}(t)[18]$ are useful.

Lemma 5. Under previous assumptions on $S(t), t \geq 0$ and $A$,

(i) $S_{\alpha}(t)$ and $T_{\alpha}(t)$ are strongly continuous;

(ii) for any $X \in H, \beta \in(0,1)$ and $\theta \in(0,1]$ one has

$$
\begin{gathered}
A T_{\alpha}(t) X=A^{1-\beta} T_{\alpha}(t) A^{\beta} X, \\
\left|A^{\theta} T_{\alpha}(t)\right| \leq \frac{\alpha C_{\theta}}{t^{\alpha \theta}} \frac{\Gamma(2-\theta)}{\Gamma(1+\alpha(1-\theta))}, \quad t \in[0, T] .
\end{gathered}
$$

Definition 6. Let $p \geq 2$ be an integer. Equation (8) is said to be stable in $p$ th moment if for arbitrarily given $\epsilon>0$ there exists a $\delta>0$ such that

$$
E\left(\sup _{t \geq 0}|X(t)|^{p}\right)<\epsilon, \quad \text { when }|\varphi|_{\mathscr{B}}<\delta .
$$

Definition 7. Let $p \geq 2$ be an integer. Equation (8) is said to be asymptotically stable in $p$ th moment if it is stable in $p$ th moment and, for any $\varphi \in \mathscr{B}_{\mathscr{F}}([m(0,0)], H)$, it holds

$$
\lim _{T \rightarrow \infty} E\left(\sup _{t \geq T}|X(t)|^{p}\right)=0 .
$$

\section{Main Result}

In this section, we prove the existence, uniqueness, and stability of the solution to fractional stochastic equation (3) by using the Banach fixed point approach.

In order to obtain the existence and stability of the solution to (3), we impose the following assumptions on (3).

(H1) There exist constants $M \geq 1$ and $a>0$ such that $|S(t)| \leq M e^{-a t}$.

(H2) There exists a positive constant $L_{1}$, for every $t \geq 0$ and $x, y \in H$, such that

$$
\begin{gathered}
|f(t, x)-f(t, y)| \leq L_{1}|x-y|, \\
|\sigma(t, x)-\sigma(t, y)| \leq L_{1}|x-y| .
\end{gathered}
$$

(H3) There exist $0<\beta<1$ such that $g$ is $H_{\beta}$-valued, $(-A)^{\beta} g$ is continuous and there exists a positive constant $M_{g}$ such that

$$
\left|(-A)^{\beta} g(t, x)-(-A)^{\beta} g(t, y)\right| \leq M_{g}|x-y|
$$

for every $t \geq 0$ and $x, y \in H$.
$(\mathrm{H} 4) \Theta=\left[4^{(p-1)}\left|(-A)^{(-\beta)}\right|^{p} M_{g}^{p}+4^{p-1} M_{g}^{p} K(\alpha, \beta)\left(T^{\alpha \beta} /\right.\right.$ $\left.\alpha \beta)^{p}+4^{p-1} \alpha^{p} M^{p} L_{1}^{p} L^{p}+4^{p-1} C_{p} \alpha^{p} M^{p} L_{1}^{p} L^{\prime(p / 2)}\right]<$ 1 , where $C_{P}=(p(p-1)) / 2^{p / 2}, M_{t}=$ $\int_{0}^{t} \theta \eta_{\alpha}(\theta) e^{-a t^{\alpha \theta}} d \theta, L=\int_{0}^{T} M_{(t-s)}(t-s)^{\alpha-1} d s$ and $L^{\prime}=\int_{0}^{T}\left[M_{t-s}(t-s)^{\alpha-1}\right]^{2} d s$.

In addition, in order to derive the stability of the solution, we further assume that

(H5)

$$
g(t, 0)=0, \quad f(t, 0)=0, \quad \sigma(t, 0)=0 .
$$

It is obvious that (3) has a trivial solution when $\varphi=0$ under the assumption (H5).

Lemma 8. Let $p \geq 2, t>0$ and let $\Phi$ be an $\mathscr{L}(K, H)$-valued, predictable process such that $E \int_{0}^{t}|\Phi(s)|_{\mathscr{L}}^{p} d s<\infty$. Then,

$$
\begin{aligned}
& \sup _{0 \leq s \leq t} E\left|\int_{0}^{s} \Phi(u) d W(u)\right|^{p} \\
& \quad \leq\left(\frac{p(p-1)}{2}\right)^{p / 2}\left(\int_{0}^{t}\left(E\left|\Phi(s)_{\mathscr{L}}^{p}\right|\right)^{2 / p}\right)^{p / 2} .
\end{aligned}
$$

Theorem 9. Let $p \geq 2$ be an integer. Assume that the conditions ( $\mathrm{H} 1)-(\mathrm{H} 4)$ hold, then the nonlinear fractional neutral stochastic differential equation (3) is asymptotically stable in the pth moment.

Proof. Denote by $\mathbb{B}$ the space of all $\mathscr{F}_{0}$-adapted process $\phi(t, w):[m(0), 0] \times \Omega \rightarrow R$, which is almost surely continuous in $t$ for fixed $w \in \Omega$ and satisfies $\phi(t, w)=\varphi(t)$ for $t \in[m(0), 0]$ and $E|\phi(t, w)|^{p} \rightarrow 0$ as $t \rightarrow 0$. It is then routine to check that $\mathbb{B}$ is a Banach space when it is equipped with a norm defined by $|\varphi|_{\mathbb{B}}=\sup _{t \geq 0} E|\varphi(t)|_{H}^{p}$. Define the nonlinear operator $\Psi: \mathbb{B} \rightarrow \mathbb{B}$ such that $(\Psi X)(t)=\varphi(t), t \in[m(0), 0]$ and, for $t \geq 0$,

$$
\begin{aligned}
(\Psi X)(t)= & S_{\alpha}(t)[\varphi(0)+g(0, \varphi)]-g(t, X(t-\tau(t))) \\
& -\int_{0}^{t}(t-s)^{\alpha-1} A T_{\alpha}(t-s) g(s, X(s-\tau(s))) d s \\
& +\int_{0}^{t}(t-s)^{\alpha-1} T_{\alpha}(t-s) f(s, X(s-\tau(s))) d s \\
& +\int_{0}^{t}(t-s)^{\alpha-1} T_{\alpha}(t-s) \sigma(s, X(s-v(s))) d W(s) .
\end{aligned}
$$

As mentioned in Luo [20], to prove the asymptotic stability it is enough to show that the operator $\Psi$ has a fixed point in $H$. To prove this result, we use the contraction mapping principle. To apply the contraction mapping principle, first we verify the mean square continuity of $\Psi$ on $[0, \infty)$. Let 
$X \in \mathbb{B}, t_{1} \geq 0$ and let $|h|$ be sufficiently small, and observe that

$$
\begin{aligned}
& E\left|(\Psi X)\left(t_{1}+h\right)-(\Psi X)\left(t_{1}\right)\right|^{p} \\
& \quad \leq 5^{p-1} \sum_{i=1}^{5} E\left|F_{i}\left(t_{1}+h\right)-F_{i}\left(t_{1}\right)\right|^{p} .
\end{aligned}
$$

Note that

$$
\begin{aligned}
& E\left|F_{1}\left(t_{1}+h\right)-F_{1}\left(t_{1}\right)\right|^{p} \\
& \quad=E\left|\left(S_{\alpha}\left(t_{1}+h\right)-S_{\alpha}\left(t_{1}\right)\right)[\varphi(0)-g(0, \varphi)]\right|^{p} .
\end{aligned}
$$

The strong continuity of $S_{\alpha}(t)[18]$ implies that the right hand of (19) goes to 0 as $|h| \rightarrow 0$. In view of Lemma 5 and the Holder's inequality, the third term of (18) becomes

$$
\begin{aligned}
& E\left|F_{3}\left(t_{1}+h\right)-F_{3}\left(t_{1}\right)\right|^{p} \\
& =E \mid \int_{0}^{t_{1}+h}\left(t_{1}+h-s\right)^{\alpha-1}(-A) T_{\alpha}\left(t_{1}+h-s\right) g \\
& \times(s, X(s-\tau(s))) d s \\
& -\int_{0}^{t_{1}}\left(t_{1}-s\right)^{\alpha-1}(-A) T_{\alpha}\left(t_{1}-s\right) g \\
& \times\left.(s, X(s-\tau(s))) d s\right|^{p} \\
& \leq 3^{p-1} E \mid \int_{t_{1}}^{t_{1}+h}\left(t_{1}+h-s\right)^{\alpha-1}(-A) T_{\alpha}\left(t_{1}+h-s\right) g \\
& \times\left.(s, X(s-\tau(s))) d s\right|^{p} \\
& +3^{p-1} E \mid \int_{0}^{t_{1}}\left[\left(t_{1}+h-s\right)^{\alpha-1}-\left(t_{1}-s\right)^{\alpha-1}\right](-A) T_{\alpha} \\
& \times\left.\left(t_{1}+h-s\right) g(s, X(s-\tau(s))) d s\right|^{p} \\
& +3^{p-1} E \mid \int_{0}^{t_{1}}\left(t_{1}-s\right)^{\alpha-1}(-A) \\
& \times\left[T_{\alpha}\left(t_{1}+h-s\right)-T_{\alpha}\left(t_{1}-s\right)\right] g \\
& \times\left.(s, X(s-\tau(s))) d s\right|^{p} \\
& \leq 3^{p-1} E\left(\int_{t_{1}}^{t_{1}+h}\left(t_{1}+h-s\right)^{\alpha-1}\left|(-A)^{1-\beta} T_{\alpha}\left(t_{1}+h-s\right)\right|\right. \\
& \left.\times\left|(-A)^{\beta} g(s, X(s-\tau(s)))\right| d s\right)^{p}
\end{aligned}
$$

$$
\begin{array}{r}
+3^{p-1} E\left(\int_{0}^{t_{1}}\left[\left(t_{1}+h-s\right)^{\alpha-1}-\left(t_{1}-s\right)^{\alpha-1}\right]\right. \\
\times\left|(-A)^{1-\beta} T_{\alpha}\left(t_{1}+h-s\right)\right| \\
\left.\times\left|(-A)^{\beta} g(s, X(s-\tau(s)))\right| d s\right)^{p} \\
+3^{p-1} E\left(\int_{0}^{t_{1}}\left(t_{1}-s\right)^{\alpha-1}\left|(-A)^{1-\beta}\right|\right.
\end{array}
$$$$
\times\left|T_{\alpha}\left(t_{1}+h-s\right)-T_{\alpha}\left(t_{1}-s\right)\right|
$$$$
\left.\times\left|(-A)^{\beta} g(s, X(s-\tau(s)))\right| d s\right)^{p}
$$$$
\leq 3^{p-1} K(\alpha, \beta) M_{g}^{p}\left(\int_{t_{1}}^{t_{1}+h}\left(t_{1}+h-s\right)^{\alpha \beta-1} d s\right)^{p-1}
$$$$
\times \int_{t_{1}}^{t_{1}+h}\left(t_{1}+h-s\right)^{\alpha \beta-1} E|X(s-\tau(s))|^{p} d s
$$$$
+3^{p-1} K(\alpha, \beta) M_{g}^{p}
$$$$
\times\left(\int_{0}^{t_{1}} \frac{\left(t_{1}+h-s\right)^{\alpha-1}-\left(t_{1}-s\right)^{\alpha-1}}{\left(t_{1}+h-s\right)^{\alpha(\beta-1)}} d s\right)^{p-1}
$$$$
\times \int_{0}^{t_{1}} \frac{\left(t_{1}+h-s\right)^{\alpha-1}-\left(t_{1}-s\right)^{\alpha-1}}{\left(t_{1}+h-s\right)^{\alpha(\beta-1)}} E|X(s-\tau(s))|^{p} d s
$$$$
+3^{p-1} \epsilon^{p} M_{g}^{p}\left|(-A)^{1-\beta}\right|^{p}\left(\int_{0}^{t_{1}}\left(t_{1}-s\right)^{\alpha-1} d s\right)^{p-1}
$$$$
\times \int_{0}^{t_{1}}\left(t_{1}-s\right)^{\alpha-1} E|X(s-\tau(s))|^{p} d s
$$$$
\leq 3^{p-1} K(\alpha, \beta) M_{g}^{p}\left(\frac{h^{\alpha \beta}}{\alpha \beta}\right)^{p-1}
$$$$
\times \int_{t_{1}}^{t_{1}+h}\left(t_{1}+h-s\right)^{\alpha \beta-1} E|X(s-\tau(s))|^{p} d s
$$$$
+3^{p-1} K(\alpha, \beta) M_{g}^{p}
$$$$
\times\left(\int_{0}^{t_{1}} \frac{\left(t_{1}+h-s\right)^{\alpha-1}-\left(t_{1}-s\right)^{\alpha-1}}{\left(t_{1}+h-s\right)^{\alpha(\beta-1)}} d s\right)^{p-1}
$$$$
\times \int_{0}^{t_{1}} \frac{\left(t_{1}+h-s\right)^{\alpha-1}-\left(t_{1}-s\right)^{\alpha-1}}{\left(t_{1}+h-s\right)^{\alpha(\beta-1)}} E|X(s-\tau(s))|^{p} d s
$$$$
+3^{p-1} \epsilon^{p} M_{g}^{p}\left|(-A)^{1-\beta}\right|^{p}\left(\frac{t_{1}^{\alpha}}{\alpha}\right)^{p-1}
$$$$
\times \int_{0}^{t_{1}}\left(t_{1}-s\right)^{\alpha-1} E|X(s-\tau(s))|^{p} d s,
$$ 
where $K(\alpha, \beta)=\alpha^{p} \Gamma_{(1+\beta)}^{p} C_{1-\beta}^{p} / \Gamma_{(1+\alpha \beta)}^{p}$. Since $\epsilon$ is sufficiently small, the right hand side of the above equation tends to zero as $|h| \rightarrow 0$.

Next we consider

$$
\begin{aligned}
& E\left|F_{4}\left(t_{1}+h\right)-F_{4}\left(t_{1}\right)\right|^{p} \\
& =E \mid \int_{0}^{t_{1}+h}\left(t_{1}+h-s\right)^{\alpha-1} T_{\alpha}\left(t_{1}+h-s\right) \\
& \times f(s, X(s-\tau(s))) d s \\
& -\left.\int_{0}^{t_{1}}\left(t_{1}-s\right)^{\alpha-1} T_{\alpha}\left(t_{1}-s\right) f(s, X(s-\tau(s))) d s\right|^{p} \\
& \leq 3^{p-1} E \mid \int_{t_{1}}^{t_{1}+h}\left(t_{1}+h-s\right)^{\alpha-1} T_{\alpha}\left(t_{1}+h-s\right) \\
& \times\left. f(s, X(s-\tau(s))) d s\right|^{p} \\
& +3^{p-1} E \mid \int_{0}^{t_{1}}\left[\left(t_{1}+h-s\right)^{\alpha-1}-\left(t_{1}-s\right)^{\alpha-1}\right] \\
& \times\left. T_{\alpha}\left(t_{1}+h-s\right) f(s, X(s-\tau(s))) d s\right|^{p} \\
& +3^{p-1} E \mid \int_{0}^{t_{1}}\left(t_{1}-s\right)^{\alpha-1}\left[T_{\alpha}\left(t_{1}+h-s\right)-T_{\alpha}\left(t_{1}-s\right)\right] f \\
& \times\left.(s, X(s-\tau(s))) d s\right|^{p} \\
& +3^{p-1} E\left(\int_{0}^{t_{1}}\left[\left(t_{1}+h-s\right)^{\alpha-1}-\left(t_{1}-s\right)^{\alpha-1}\right]\right. \\
& \left.\times\left|T_{\alpha}\left(t_{1}+h-s\right)\right||f(s, X(s-\tau(s)))| d s\right)^{p} \\
& +3^{p-1} E\left(\int_{0}^{t_{1}}\left(t_{1}-s\right)^{\alpha-1}\left|T_{\alpha}\left(t_{1}+h-s\right)-T_{\alpha}\left(t_{1}-s\right)\right|\right. \\
& \times|f(s, X(s-\tau(s)))| d s)^{p} .
\end{aligned}
$$

By the Holder's inequality, we obtain

$$
\begin{aligned}
\leq & 3^{p-1} L_{1}^{p} M^{p} \alpha^{p}\left(\int_{t_{1}}^{t_{1}+h} M_{t_{1}+h-s}\left(t_{1}+h-s\right)^{\alpha-1} d s\right)^{p-1} \\
& \times \int_{t_{1}}^{t_{1}+h} M_{t_{1}+h-s}\left(t_{1}+h-s\right)^{\alpha-1} E|X(s-\tau(s))|^{p} d s \\
& +3^{p-1} L_{1}^{p} M^{p} \alpha^{p} \\
& \times\left(\int_{0}^{t_{1}} M_{t_{1}+h-s}\left[\left(t_{1}+h-s\right)^{\alpha-1}-\left(t_{1}-s\right)^{\alpha-1}\right] d s\right)^{p-1}
\end{aligned}
$$

$$
\begin{aligned}
& \times \int_{0}^{t_{1}} M_{t_{1}+h-s}\left[\left(t_{1}+h-s\right)^{\alpha-1}-\left(t_{1}-s\right)^{\alpha-1}\right] E \\
& \quad \times|X(s-\tau(s))|^{p} d s \\
& +3^{p-1} \epsilon^{p} L_{1}^{p}\left(\frac{t_{1}^{\alpha}}{\alpha}\right)^{p-1} \\
& \times \int_{0}^{t_{1}}\left(t_{1}-s\right)^{\alpha-1} E|X(s-\tau(s))|^{p} d s .
\end{aligned}
$$

Therefore, the right hand side of the above equation tends to zero as $|h| \rightarrow 0$ and $\epsilon$ sufficiently small. Further, we have

$$
\begin{aligned}
& E\left|F_{5}\left(t_{1}+h\right)-F_{5}\left(t_{1}\right)\right|^{p} \\
& =E \mid \int_{0}^{t_{1}+h}\left(t_{1}+h-s\right)^{\alpha-1} T_{\alpha}\left(t_{1}+h-s\right) \\
& \times \sigma(s, X(s-\tau(s))) d W(s) \\
& -\int_{0}^{t_{1}}\left(t_{1}-s\right)^{\alpha-1} T_{\alpha}\left(t_{1}-s\right) \\
& \times\left.\sigma(s, X(s-\tau(s))) d W(s)\right|^{p} \\
& \leq 3^{p-1} E \mid \int_{t_{1}}^{t_{1}+h}\left(t_{1}+h-s\right)^{\alpha-1} T_{\alpha}\left(t_{1}+h-s\right) \\
& \times\left.\sigma(s, X(s-\tau(s))) d W(s)\right|^{p} \\
& +3^{p-1} E \mid \int_{0}^{t_{1}}\left[\left(t_{1}+h-s\right)^{\alpha-1}-\left(t_{1}-s\right)^{\alpha-1}\right] \\
& \times\left. T_{\alpha}\left(t_{1}+h-s\right) \sigma(s, X(s-\tau(s))) d W(s)\right|^{p} \\
& +3^{p-1} E \mid \int_{0}^{t_{1}}\left(t_{1}-s\right)^{\alpha-1}\left[T_{\alpha}\left(t_{1}+h-s\right)-T_{\alpha}\left(t_{1}-s\right)\right] \\
& \times\left.\sigma(s, X(s-\tau(s))) d W(s)\right|^{p} \\
& \leq 3^{p-1} C_{p} E\left(\int_{t_{1}}^{t_{1}+h}\left(t_{1}+h-s\right)^{2(\alpha-1)}\left|T_{\alpha}\left(t_{1}+h-s\right)\right|^{2}\right. \\
& \left.\times|\sigma(s, X(s-\tau(s)))|^{2} d s\right)^{p / 2} \\
& +3^{p-1} C_{p} E\left(\int_{0}^{t_{1}}\left[\left(t_{1}+h-s\right)^{\alpha-1}-\left(t_{1}-s\right)^{\alpha-1}\right]^{2}\right. \\
& \times\left|T_{\alpha}\left(t_{1}+h-s\right)\right|^{2} \\
& \left.\times|\sigma(s, X(s-\tau(s)))|^{2} d s\right)^{p / 2}
\end{aligned}
$$




$$
\begin{aligned}
& +3^{p-1} C_{p} E\left(\int_{0}^{t_{1}}\left(t_{1}-s\right)^{2(\alpha-1)}\right. \\
& \times\left|T_{\alpha}\left(t_{1}+h-s\right)-T_{\alpha}\left(t_{1}-s\right)\right|^{2} \\
& \times 3^{p-1} C_{p} L_{1}^{p} M^{p} \alpha^{p} \\
& \times\left(\int_{t_{1}}^{t_{1}+h}\left(M_{t_{1}+h-s}\left(t_{1}+h-s\right)^{\alpha-1}\right)^{p /(p-2)} d s\right)^{(p-2) / 2} \\
& \left.\left.\times \int_{t_{1}}^{t_{1}+h}\left(M_{t_{1}+h-s}\left(t_{1}+h-s\right)^{\alpha-1}\right)^{p / 2}(s)\right)\left.\right|^{2} d s\right)^{t_{1}}\left(t_{1}-s\right)^{p(\alpha-1) / 2} E|X(s-\tau(s))|^{p} d s . \\
& \times \int_{0}^{t_{1}}\left(M_{t_{1}+h-s}\left[\left(t_{1}+h-s\right)^{\alpha-1}-\left(t_{1}-s\right)^{\alpha-1}\right]\right)^{p / 2} \\
& \times E|X(s-\tau(s))|^{p} d s+3^{p-1} C_{p} L_{1}^{p} M^{p} \alpha^{p} \\
& \times\left(\int _ { 0 } ^ { t _ { 1 } } \left(M _ { t _ { 1 } + h - s } \left[\left(t_{1}+h-s\right)^{\alpha-1}\right.\right.\right. \\
& \times E|X(s-\tau(s))|^{p} d s \\
& \left.\left.\left.\quad+\left(t_{1}-s\right)^{\alpha-1}\right]\right)^{p /(p-2)} d s\right)^{(p-2) / 2}
\end{aligned}
$$

As above, the right hand side of the above inequality tends to zero. Similarly, we have $F_{2} \rightarrow 0$ as $h \rightarrow 0$. Thus $\Psi$ is continuous in $p$ th moment on $[0, \infty)$.

Next we show that $\Psi(\mathbb{B}) \in \mathbb{B}$. Let $X \in \mathbb{B}$. From (18), we have

$$
\begin{gathered}
E|(\Psi X)(t)|^{p} \\
\leq 6^{p-1} E\left|S_{\alpha}(t) \varphi(0)\right|^{p}+6^{p-1} E\left|S_{\alpha}(t) g(0, \varphi)\right|^{p} \\
+6^{p-1} E|g(t, X(t-\tau(t)))|^{p} \\
+6^{p-1} E \mid \int_{0}^{t}(t-s)^{\alpha-1}(-A) T_{\alpha}(t-s) \\
\quad \times\left. g(s, X(s-\tau(s))) d s\right|^{p}
\end{gathered}
$$

$$
\begin{gathered}
+6^{p-1} E\left|\int_{0}^{t}(t-s)^{\alpha-1} T_{\alpha}(t-s) f(s, X(s-\tau(s))) d s\right|^{p} \\
+6^{p-1} E \mid \int_{0}^{t}(t-s)^{\alpha-1} T_{\alpha}(t-s) \\
\times\left.\sigma(s, X(s-v(s))) d W(s)\right|^{p} .
\end{gathered}
$$

Now, we estimate the terms on the right hand side of (24) by using the assumptions (H1), (H3), and (H4). Now, we have

$$
\begin{aligned}
& 6^{p-1} E\left|S_{\alpha}(t) \varphi(0)\right|^{p} \\
& \leq 6^{p-1} M^{p}\left(\int_{0}^{\infty} \eta_{\alpha}(\theta) e^{-\alpha t^{\alpha} \theta} d \theta\right)^{p}|\varphi|_{\mathscr{B}}^{p} \\
& \longrightarrow 0 \quad \text { as } t \longrightarrow \infty \\
& 6^{p-1} E\left|S_{\alpha}(t) g(0, \varphi)\right|^{p} \\
& \leq 6^{p-1} M^{p}\left(\int_{0}^{\infty} \eta_{\alpha}(\theta) e^{-\alpha t^{\alpha} \theta} d \theta\right)^{p}\left|(-A)^{-\beta}\right|^{p} M_{g}^{p}|\varphi|_{\mathscr{B}}^{p} \\
& \longrightarrow 0 \quad \text { as } t \longrightarrow \infty, \\
& 6^{p-1} E|g(t, X(t-\tau(t)))|^{p} \\
& \leq 6^{p-1}\left|(-A)^{-\beta}\right|^{p} M_{g}^{p} E|X(t-\tau(t))|^{p} .
\end{aligned}
$$

For $X(t) \in \mathbb{B}$ and for any $\epsilon>0$ there exists a $t_{1}>0$ such that $E|X(t-\tau(t))|^{p} \leq \epsilon$ for $t \geq t_{1}$.

Therefore,

$$
6^{p-1} E|g(t, X(t-\tau(t)))|^{p} \longrightarrow 0 \quad \text { as } t \longrightarrow \infty .
$$

For the fourth term of (24), we have

$$
\begin{aligned}
& 6^{p-1} E\left|\int_{0}^{t}(t-s)^{\alpha-1}(-A) T_{\alpha}(t-s) g(s, X(s-\tau(s))) d s\right|^{p} \\
& \leq 6^{p-1} E\left(\int_{0}^{t}(t-s)^{\alpha-1}\left|(-A)^{1-\beta} T_{\alpha}(t-s)\right|\right. \\
& \left.\quad \times\left|(-A)^{\beta} g(s, X(s-\tau(s)))\right| d s\right)^{p} \\
& \leq 6^{p-1} M_{g}^{p} K(\alpha, \beta)\left(\int_{0}^{t}(t-s)^{\alpha \beta-1} d s\right)^{p-1} \\
& \quad \times \int_{0}^{t}(t-s)^{\alpha \beta-1} E|X(s-\tau(s))|^{p} d s \\
& \leq 6^{p-1} M_{g}^{p} K(\alpha, \beta)\left(\frac{T^{\alpha \beta}}{\alpha \beta}\right)^{p-1} \\
& \quad \times \int_{0}^{t}(t-s)^{\alpha \beta-1} E|X(s-\tau(s))|^{p} d s \longrightarrow 0 \quad \text { as } t \longrightarrow \infty .
\end{aligned}
$$


Also, we have

$$
\begin{aligned}
& 6^{p-1} E\left|\int_{0}^{t}(t-s)^{\alpha-1} T_{\alpha}(t-s) f(s, X(s-\tau(s))) d s\right| \\
& \leq 6^{p-1} E\left(\int_{0}^{t}(t-s)^{\alpha-1}\left|T_{\alpha}(t-s)\right|\right. \\
& \quad \times|f(s, X(s-\tau(s)))| d s)^{p} \\
& \leq 6^{p-1} \alpha^{p} M^{p} L_{1}^{p}\left(\int_{0}^{t} M_{t-s}(t-s)^{\alpha-1} d s\right)^{p-1} \\
& \left.\quad \times \int_{0}^{t} M_{t-s}(t-s)^{\alpha-1} E \mid X(s-\tau(s))\right)\left.\right|^{p} d s, \\
& 6^{p-1} E\left|\int_{0}^{t}(t-s)^{\alpha-1} T_{\alpha}(t-s) \sigma(s, X(s-v(s))) d W(s)\right|^{p} \\
& \leq\left. 6^{p-1} C_{p} E\left|\int_{0}^{t}(t-s)^{2(\alpha-1)}\right| T_{\alpha}(t-s)\right|^{2} \\
& \quad \times \int_{0}^{t} M_{t-s}(t-s)^{\alpha-1} E|X(s-v(s))|^{p} d s . \\
& \times\left.|\sigma(s, X(s-v(s)))|^{2} d s\right|^{p / 2}
\end{aligned}
$$

By the same discussion as above, we have that (28) tends to zero as $t \rightarrow \infty$. Thus $E|\Psi X(t)|^{p} \rightarrow 0$ as $t \rightarrow \infty$. We conclude that $\Psi(\mathbb{B}) \in \mathbb{B}$.

Finally, we prove that $\Psi$ has a unique fixed point. Indeed, for any $X, Y \in \mathbb{B}$, we have

$$
\begin{aligned}
& \sup _{t \in[0, T]} E|(\Psi X)(t)-(\Psi Y)(t)|^{p} \\
& \leq 4^{p-1} \sup _{t \in[0, T]} E|g(t, X(t-\tau(t)))-g(t, Y(t-\tau(t)))|^{p} \\
& +4^{p-1} \sup _{t \in[0, T]} E \mid \int_{0}^{t}(t-s)^{\alpha-1}(-A) T_{\alpha}(t-s) \\
& \times[g(s, X(s-\tau(s))) \\
& +4^{p-1} \sup _{t \in[0, T]} E \mid \int_{0}^{t}(t-s)^{\alpha-1} T_{\alpha}(t-s) \\
& \times[f(s, X(s-\tau(s))) \\
& \quad-g(s, Y(s-\tau(s)))]\left.d s\right|^{p} \\
& \quad-f(s, Y(s-\tau(s)))]\left.d s\right|^{p}
\end{aligned}
$$

$$
\begin{array}{r}
+4^{p-1} \sup _{t \in[0, T]} E \mid \int_{0}^{t}(t-s)^{\alpha-1} T_{\alpha}(t-s) \\
\times[\sigma(s, X(s-v(s))) \\
\quad-\sigma(s, Y(s-v(s)))]\left.d W(s)\right|^{p}
\end{array}
$$$$
\leq 4^{p-1}\left|(-A)^{-\beta}\right|^{p} M_{g}^{p} \sup _{t \in[0, T]} E|X(t)-Y(t)|^{p}
$$$$
+4^{p-1} \sup _{t \in[0, T]} E\left(\int_{0}^{t}(t-s)^{\alpha-1}\left|(-A)^{1-\beta} T_{\alpha}(t-s)\right|\right.
$$$$
\times \mid(-A)^{\beta}[g(s, X(s-\tau(s)))
$$$$
-g(s, Y(s-\tau(s)))] \mid d s)^{p}
$$$$
+4^{p-1} \sup _{t \in[0, T]} E\left(\int_{0}^{t}(t-s)^{\alpha-1}\left|T_{\alpha}(t-s)\right|\right.
$$$$
\times \mid f(s, X(s-\tau(s)))
$$$$
-f(s, Y(s-\tau(s))) \mid d s)^{p}
$$$$
+4^{p-1} C_{p} \sup _{t \in[0, T]} E\left(\int_{0}^{t}(t-s)^{2(\alpha-1)}\left|T_{\alpha}(t-s)\right|^{2}\right.
$$$$
\times \mid \sigma(s, X(s-v(s)))
$$

$$
\begin{array}{r}
\left.-\left.\sigma(s, Y(s-v(s)))\right|^{2} d s\right)^{p / 2} \\
\leq\left[4^{p-1}\left|(-A)^{-\beta}\right|^{p} M_{g}^{p}+4^{p-1} M_{g}^{p} K(\alpha, \beta)\left(\frac{T^{\alpha \beta}}{\alpha \beta}\right)^{p}\right. \\
+4^{p-1} \alpha^{p} M^{p} L_{1}^{p} L^{p} \\
\left.+4^{p-1} C_{p} \alpha^{p} M^{p} L_{1}^{p} L^{\prime p / 2}\right] \sup _{t \in[0, T]} E|X(t)-Y(t)|^{p} .
\end{array}
$$

Therefore, $\Psi$ is a contradiction mapping and hence there exists a unique fixed point, which is a mild solution of (3) with $X(s)=\varphi(s)$ on $[m(0), 0]$ and $E|X(t)|^{p} \rightarrow 0$ as $t \rightarrow \infty$.

To show the asymptotic stability of the mild solution of (3), as the first step, we have to prove the stability in $p$ th moment. Let $\epsilon>0$ be given and choose $\delta>0$ such that $\delta<\epsilon$ satisfies $6^{p-1}\left[M^{p}+M^{p}\left|(-A)^{-\beta}\right|^{p} M_{g}^{p}\right] \delta+6^{p-1}\left[\left|(-A)^{-\beta}\right|^{p} M_{g}^{p}+\right.$ $\left.M_{g}^{p} K(\alpha, \beta)\left(T^{\alpha \beta} / \alpha \beta\right)^{p}+\alpha^{p} M^{p} L_{1}^{p} L^{p}+C_{p} \alpha^{p} M^{p} L_{1}^{p} L^{\prime p / 2}\right] \epsilon<$ $\epsilon$.

If $X(t)=X(t, \varphi)$ is mild solution of (3), with $|\varphi|_{\mathscr{B}}^{p}<\delta$, then $(\Psi X)(t)=X(t)$ satisfies $E|X(t)|^{p}<\epsilon$ for every $t \geq 0$. Notice that $E|X(t)|^{p}<\epsilon$ on $t \in[m(0), 0]$. If there exists $\bar{t}$ 
such that $E|X(\bar{t})|^{p}=\epsilon$ and $E|X(s)|^{p}<\epsilon$ for $s \in[m(0), \bar{t}]$. Then (24) show that

$$
\begin{gathered}
E|X(\bar{t})|^{p} \\
\leq 6^{p-1}\left[M^{p}\left(\eta_{\alpha}(\theta) e^{-\alpha \bar{t}^{\alpha} \theta}\right)^{p}\right. \\
\left.+M^{p}\left(\eta_{\alpha}(\theta) e^{-\alpha \bar{t} \theta}\right)^{p}\left|(-A)^{-\beta}\right|^{p} M_{g}^{p}\right] \delta \\
+6^{p-1}\left[\left|(-A)^{-\beta}\right|^{p} M_{g}^{p}+M_{g}^{p} K(\alpha, \beta)\left(\frac{T^{\alpha \beta}}{\alpha \beta}\right)^{p}\right. \\
\left.\quad+\alpha^{p} M^{p} L_{1}^{p} L^{p}+C_{p} \alpha^{p} M^{p} L_{1}^{p} L^{\prime p / 2}\right] \epsilon \\
<\epsilon \quad
\end{gathered}
$$

which contradicts the definition of $\bar{t}$. Therefore, the mild solution of (3) is asymptotically stable in pth moment.

In particular, when $p=2$ from Theorem 9 we have the following.

Theorem 10. Suppose that the conditions (H1)-(H3) hold. Then, the stochastic fractional differential equations (3) are mean square asymptotically stable if $4 M_{g}^{2}\left[\left|(-A)^{-\beta}\right|^{2}+\right.$ $\left.V(\alpha, \beta)\left(T^{\alpha \beta} / \alpha \beta\right)^{2}\right]+4 \alpha^{2} M^{2} L_{1}^{2}\left[L^{2}+L^{\prime}\right]<1$, where $V(\alpha, \beta)=$ $\alpha^{2} \Gamma_{(1+\beta)}^{2} C_{1-\beta}^{2} / \Gamma_{(1+\alpha \beta)}^{2}$.

When $g \equiv 0, p=2$, (3) reduces to

$$
\begin{gathered}
{ }^{c} D_{t}^{\alpha} X(t)=A X(t)+f(t, X(t-\tau(t))) \\
+\sigma(t, X(t-v(t))) \frac{d W(t)}{d t}, \quad t \geq 0, \\
X_{0}(\cdot)=\varphi \in \mathscr{B}_{\mathscr{F}}([m(0), 0], H) .
\end{gathered}
$$

From Theorems 9 and 10, we can easily get the following result.

Corollary 11. Suppose the assumptions (H1) and (H2) hold. Then, the stochastic equations (8) are mean square asymptotically stable if $2 \alpha^{2} M^{2} L_{1}^{2}\left[L^{2}+L^{\prime}\right]<1$.

Example 12. Consider the following stochastic nonlinear fractional partial differential equation with infinite delay in the following form

$$
\begin{gathered}
{ }^{c} D_{t}^{\alpha}[u(t, y)+\widehat{g}(t, u(t-\tau, y)] \\
=\frac{\partial^{2} u(t, y)}{\partial y^{2}}+\widehat{f}(t, u(t-\tau, y)) \\
+\widehat{\sigma}(t, u(t-\tau, y)) \frac{d W(t)}{d t}, \\
u(t, 0)=u(t, \pi)=0, \\
u(t, y)=\phi(t, y), \quad y \in[0, \pi], t \leq 0,
\end{gathered}
$$

where $W(t)$ denotes a standard cylindrical Wiener process and a standard one-dimensional Brownian motion. To write the system (32) into the abstract form of (3), we consider the space $H=K=L^{2}[0, \pi]$ and define the operator $A: D(A) \subset$ $H \rightarrow H$ by $A w=w^{\prime \prime}$ with domain

$$
\begin{gathered}
D(A)=\left\{w \in X ; w, w^{\prime}\right. \text { are absolutely continuous, } \\
\left.w^{\prime \prime} \in X, w(0)=w(\pi)=0\right\}, \\
A w=\sum_{n=1}^{\infty} n^{2}\left(w, w_{n}\right) w_{n}, \quad w \in D(A),
\end{gathered}
$$

where $w_{n}(s)=\sqrt{2} \sin (n s), n=1,2, \ldots$ is the orthogonal set of eigenvectors in $A$. It is well known that $A$ generates a compact, analytic semigroup $\{S(t), t \geq 0\}$ in $X$ and

$$
S(t) w=\sum_{n=1}^{\infty} e^{-n^{2} t}\left(w, w_{n}\right) w_{n} .
$$

It is well known that $|S(t)| \leq e^{-\pi^{2} t}$. Take $p=2$. Since $M=1$, we can get the inequality $4\left[\left|(-A)^{-\beta}\right|^{2}+V(\alpha, \beta)\left(\pi^{\alpha \beta} / \alpha \beta\right)^{2}+\right.$ $\left.\alpha^{2}\left(L^{2}+L^{\prime}\right)\right]<1$. Further, if we impose suitable conditions on $\widehat{g}, \widehat{f}$, and $\widehat{\sigma}$ to verify assumptions of Theorem 10 , then we can conclude that the mild solution of (32) is mean square asymptotically stable.

\section{References}

[1] J. Bao, Z. Hou, and C. Yuan, "Stability in distribution of mild solutions to stochastic partial differential equations," Proceedings of the American Mathematical Society, vol. 138, no. 6, pp. 2169-2180, 2010.

[2] M. M. Fu and Z. X. Liu, "Square-mean almost automorphic solutions for some stochastic differential equations," Proceedings of the American Mathematical Society, vol. 138, no. 10, pp. 3689-3701, 2010.

[3] Y.-K. Chang, Z.-H. Zhao, G. M. N’Guérékata, and R. Ma, "Stepanov-like almost automorphy for stochastic processes and applications to stochastic differential equations," Nonlinear Analysis: Real World Applications, vol. 12, no. 2, pp. 1130-1139, 2011.

[4] Y.-K. Chang, Z.-H. Zhao, and G. M. N’Guérékata, “A new composition theorem for square-mean almost automorphic functions and applications to stochastic differential equations," Nonlinear Analysis. Theory, Methods and Applications A, vol. 74, no. 6, pp. 2210-2219, 2011.

[5] Y.-K. Chang, Z.-H. Zhao, and G. M. N’Guérékata, “Squaremean almost automorphic mild solutions to non-autonomous stochastic differential equations in Hilbert spaces," Computers and Mathematics with Applications, vol. 61, no. 2, pp. 384-391, 2011.

[6] R. Hilfer, Applications of Fractional Calculus in Physics, World Scientific Publishing, Singapore, 2000.

[7] M. M. El-Borai, K. EI-Said EI-Nadi, and H. A. Fouad, "On some fractional stochastic delay differential equations," Computers and Mathematics with Applications, vol. 59, no. 3, pp. 1165-1170, 2010. 
[8] H. Chen, "Impulsive-integral inequality and exponential stability for stochastic partial differential equations with delays," Statistics and Probability Letters, vol. 80, no. 1, pp. 50-56, 2010.

[9] M. M. El-Borai, O. L. Moustafa, and H. M. Ahmed, "Asymptotic stability of some stochastic evolution equations," Applied Mathematics and Computation, vol. 144, no. 2-3, pp. 273-286, 2003.

[10] T. Caraballo and K. Liu, "Exponential stability of mild solutions of stochastic partial differential equations with delays," Stochastic Analysis and Applications, vol. 17, no. 5, pp. 743-763, 1999.

[11] L. Wan and J. Duan, "Exponential stability of non-autonomous stochastic partial differential equations with finite memory," Statistics and Probability Letters, vol. 78, no. 5, pp. 490-498, 2008.

[12] R. Sakthivel, Y. Ren, and H. Kim, "Asymptotic stability of second-order neutral stochastic differential equations," Journal of Mathematical Physics, vol. 51, no. 5, article 005005, pp. 1-9, 2010.

[13] R. Sakthivel and J. Luo, "Asymptotic stability of nonlinear impulsive stochastic differential equations," Statistics and Probability Letters, vol. 79, no. 9, pp. 1219-1223, 2009.

[14] R. Sakthivel and J. Luo, "Asymptotic stability of impulsive stochastic partial differential equations with infinite delays," Journal of Mathematical Analysis and Applications, vol. 356, no. 1, pp. 1-6, 2009.

[15] D. Zhao and D. Han, "Mean square exponential and nonexponential asymptotic stability of impulsive stochastic Volterra equations," Journal of Inequalities and Applications, vol. 2011, article 9, 2011.

[16] M. M. El-Borai, K. E.-S. El-Nadi, O. L. Mostafa, and H. M. Ahmed, "Volterra equations with fractional stochastic integrals," Mathematical Problems in Engineering, vol. 2004, no. 5, pp. 453-468, 2004.

[17] H. M. Ahmed, "Controllability of fractional stochastic delay equations," Lobachevskii Journal of Mathematics, vol. 30, no. 3, pp. 195-202, 2009.

[18] Y. Zhou and F. Jiao, "Existence of mild solutions for fractional neutral evolution equations," Computers and Mathematics with Applications, vol. 59, no. 3, pp. 1063-1077, 2010.

[19] I. Podlubny, Fractional Differential Equations, Academic Press, San Diego, Calif, USA, 1999.

[20] J. Luo, "Fixed points and exponential stability of mild solutions of stochastic partial differential equations with delays," Journal of Mathematical Analysis and Applications, vol. 342, no. 2, pp. 753-760, 2008.

[21] I. Podlubny, Fractional Differential Equations, Academic Press, San Diego, Calif, USA, 1999. 


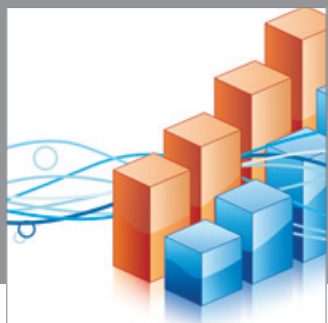

Advances in

Operations Research

mansans

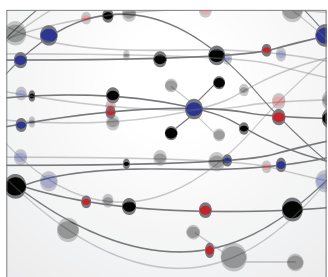

The Scientific World Journal
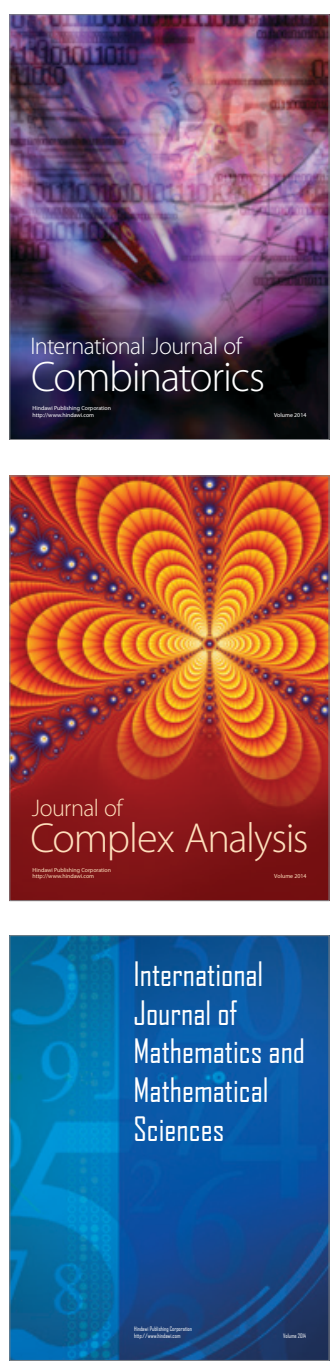
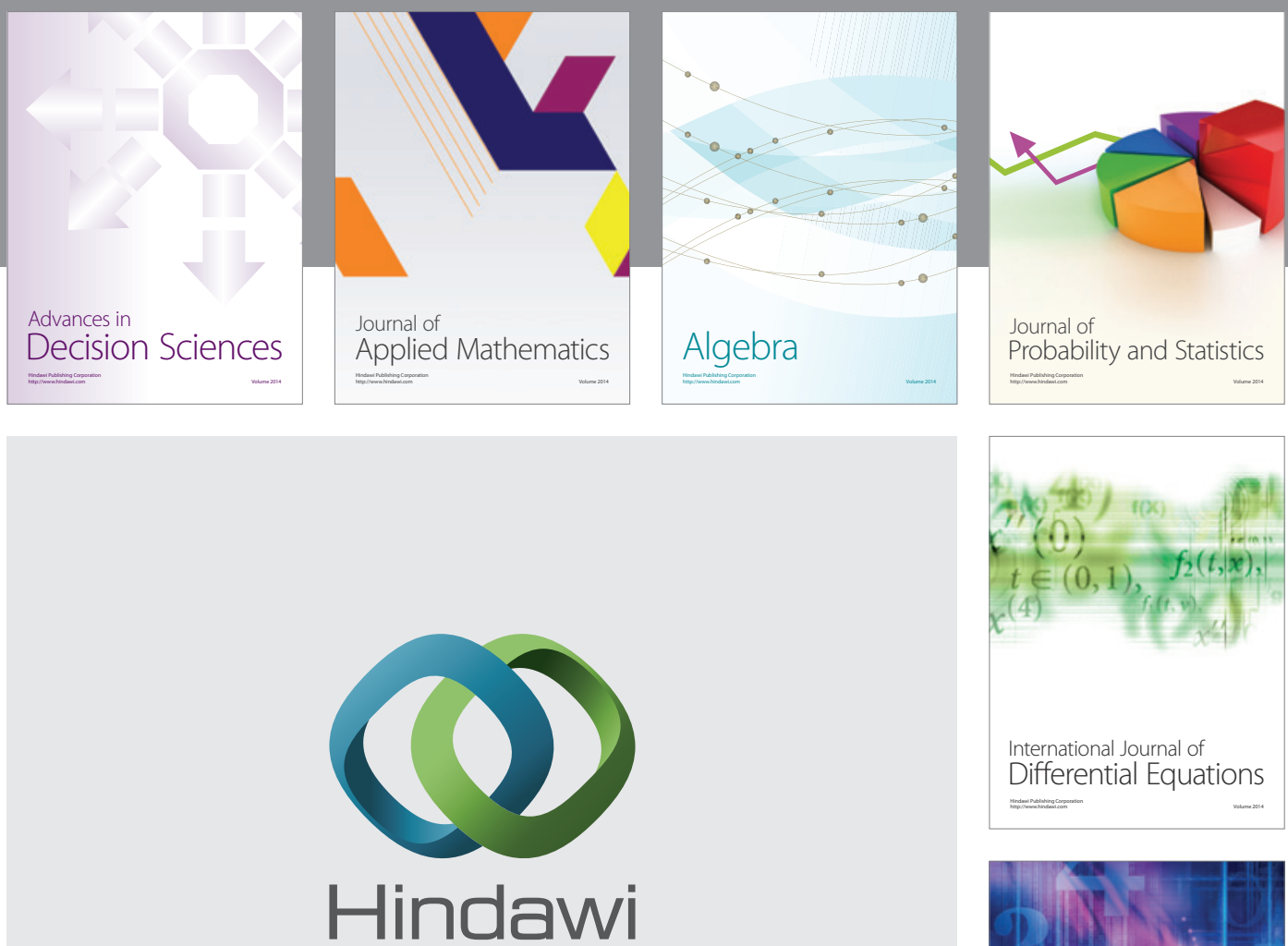

Submit your manuscripts at http://www.hindawi.com
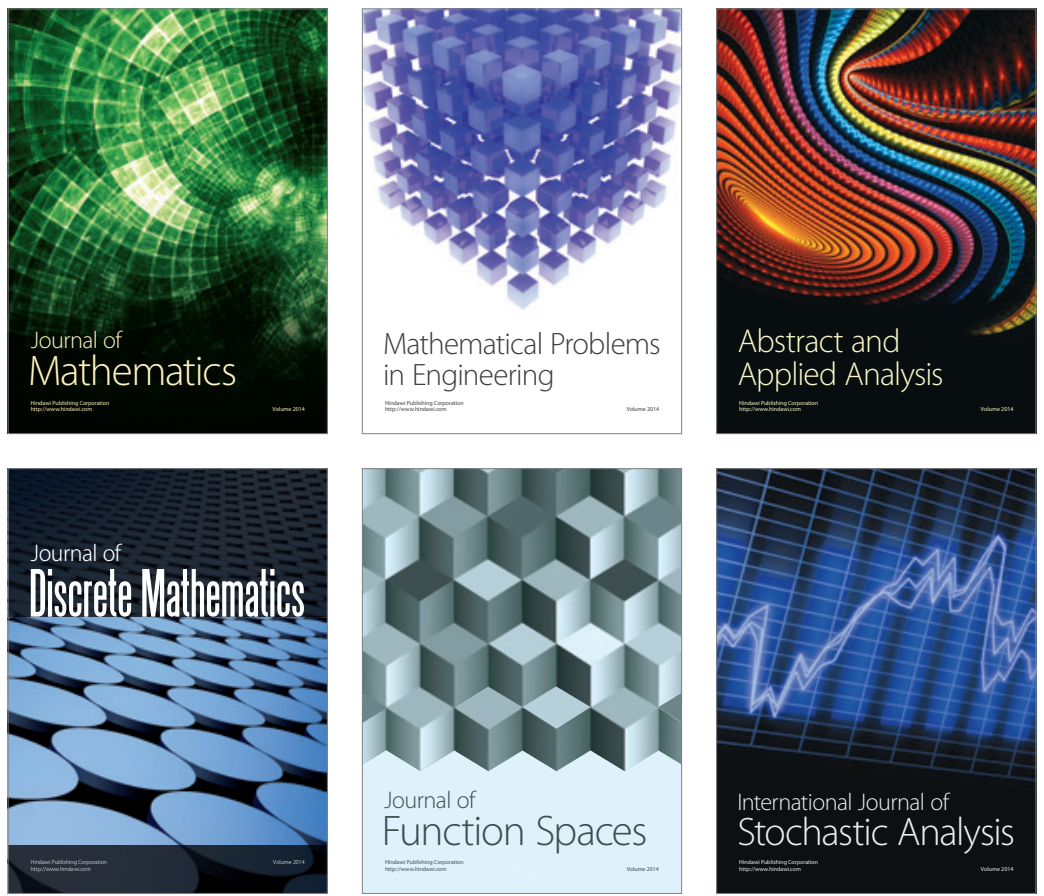

Journal of

Function Spaces

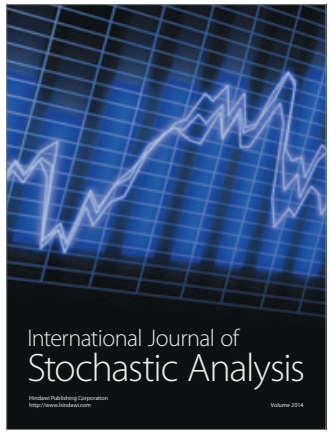

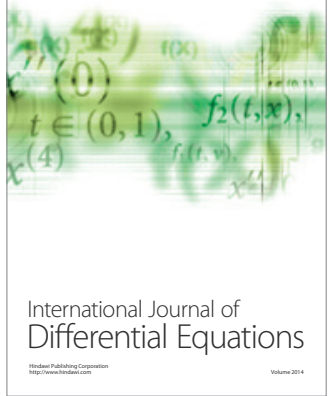
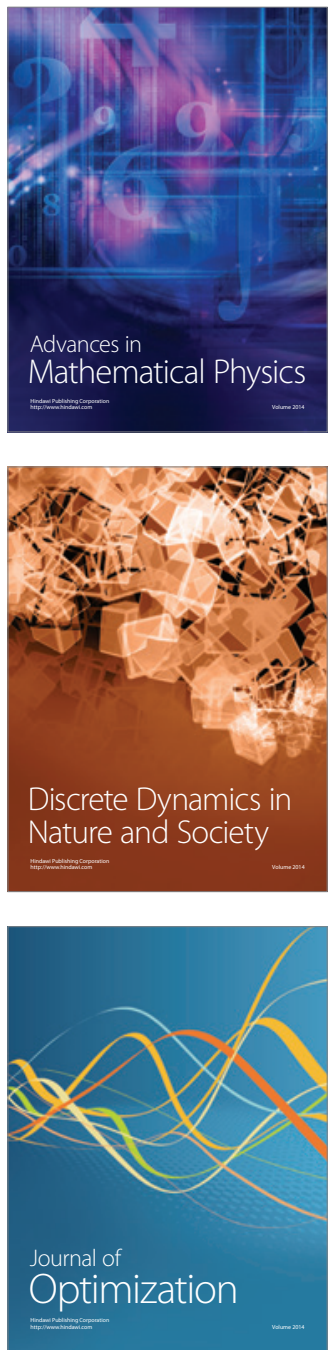